\title{
Anti-VEGF therapy for central retinal vein occlusion caused by tuberculosis-associated uveitis: a case report
}

This article was published in the following Dove Press journal:

International Medical Case Reports Journal

I8 April 2017

Number of times this article has been viewed

\section{Manzo Taguchi \\ Yutaka Sakurai \\ Takayuki Kanda \\ Masaru Takeuchi}

Department of Ophthalmology, National Defense Medical College, Tokorozawa, Saitama, Japan
Correspondence: Masaru Takeuchi Department of Ophthalmology, National Defense Medical College, 3-2 Namiki, Tokorozawa City, Saitama 359-85I3, Japan

Tel +8I 42995 I5II

Fax +8I 429935332

Email masatake@ndmc.ac.jp
Background: Tuberculosis (TB)-associated uveitis presents periphlebitis, occasionally causing central retinal vascular occlusion (CRVO). Intravitreal injection of ranibizumab (IVR) is an effective treatment for CRVO, which improves macular edema (ME) by reducing vascular permeability and prevents progression of retinal nonperfusion in CRVO. We report a case of CRVO due to TB-associated uveitis, which initially remitted by repeated IVR as an adjunct to anti-TB therapy and systemic corticosteroids, but subsequently led to severe vitreous hemorrhage (VH). Case presentation: A 28-year-old man was referred to our hospital with a 2-week history of uveitis in his right eye. Ophthalmoscopic examination of the right eye revealed fine keratoprecipitates and moderate cell infiltration into the anterior chamber and vitreous. No obvious retinal lesion was observed. Despite initiation of topical corticosteroids, CRVO developed a few weeks later in the right eye. TB-associated uveitis was diagnosed based on a positive tuberculin skin test and interferon- $\gamma$ release assay in addition to the ocular findings. Anti-TB therapy together with IVR and systemic corticosteroids was initiated. Although fundus findings associated with CRVO gradually improved, CRVO with VH recurred before the fourth IVR. Although IVR was continued, VH progressed to obscure fundus observation. Therefore, vitrectomy and panretinal photocoagulation were performed. After surgery, ocular inflammation was controlled, and antiTB therapy was continued for 6 months and was suspended.

Conclusion: In addition to anti-TB therapy with or without corticosteroids, panretinal photocoagulation for retinal nonperfusion area in TB-associated uveitis should be performed for preventing neovascularization that may cause $\mathrm{VH}$, and this role of panretinal photocoagulation cannot be replaced by anti-VEGF therapy.

Keywords: tuberculosis, uveitis, VEGF, ranibizumab, central retinal vascular occlusion

\section{Background}

Tuberculosis (TB) causes active uveitis, and the suggestive clinical signs include iridocyclitis with posterior synechiae, retinal perivasculitis often with capillary nonperfusion, multifocal serpiginoid choroiditis, choroidal or optic disc granuloma(s), and optic neuropathy. Diagnosis of TB-associated uveitis is established by combination of tuberculous skin testing (TST), an interferon- $\gamma$ release assay (IGRA), or chest $\mathrm{X}$-ray or computed tomography (CT) in addition to the ocular clinical features. Central retinal vascular occlusion (CRVO) is rare in young adults, but occasionally occurs by perivasculitis of TB-associated uveitis. ${ }^{1-3}$ Ranibizumab is a recombinant, humanized, monoclonal antibody that neutralizes all active forms of vascular endothelial growth factor (VEGF) A. Intravitreal injection of ranibizumab (IVR) is an effective treatment for CRVO, which improves macular edema (ME) by reducing vascular permeability and 
prevents progression of retinal nonperfusion in CRVO. ${ }^{4} \mathrm{We}$ report a case of CRVO due to TB-associated uveitis, which initially remitted by repeated IVR as an adjunct to anti-TB therapy and systemic corticosteroids, but subsequently led to severe vitreous hemorrhage $(\mathrm{VH})$.

\section{Case presentation}

A 28-year-old man was referred to our hospital with a 2-week history of uveitis in his right eye. The best corrective visual acuity (BCVA) was 20/25 and ocular pressure was within normal range. Ophthalmoscopic examination of the right eye revealed fine keratoprecipitates and moderate cell infiltration into the anterior chamber and vitreous. No obvious retinal lesion was observed. However, fundus fluorescein angiography (FA) showed hyperfluorescence in the optic disc and a petaloid pattern of fluorescence pooling in the macula indicating cystoid ME. Despite initiation of topical corticosteroids, optic disc swelling, extensive preretinal and intraretinal hemorrhages developed a few weeks later. Vascular tortuosity associated with CRVO was observed in the right eye, and BCVA decreased to 2/200 due to ME and hemorrhage (Figure 1A). No systemic symptoms associated with Behçet's disease and no laboratory tests or chest CT scans suggesting sarcoidosis were found. Although there was no family history suggestive of TB and systemic source of TB infection was not identified on physical examination, TB-associated uveitis was diagnosed based on a positive TST and IGRA in addition to the ocular findings. Anti-TB therapy with rifampin, isoniazid, pyrazinamide, and ethambutol, together with monthly IVR and systemic corticosteroids $(0.5 \mathrm{mg} / \mathrm{kg})$, was initiated. After two IVR sessions, fundus findings associated with CRVO improved markedly, and
ME was resolved except for persistent serous retinal detachment (Figure 1B). BCVA recovered to 20/25. However, before the fourth IVR session, severe retinal hemorrhage on the nasal side and $\mathrm{VH}$ were found in the right eye, with exacerbation of ME (Figure 2A). Although FA was required to evaluate nonperfusion area and the activity of ocular inflammation, it could not be performed since pruritus and nausea were observed at the first FA. In spite of continuation of IVR, VH progressed to obscure fundus observation, and BCVA decreased to hand motion. Therefore, vitrectomy and panretinal photocoagulation were performed. After surgery, iridocyclitis was observed, but it was controlled by topical corticosteroid, and anti-TB therapy was continued for 6 months and was suspended. Since a second vitrectomy with cataract surgery was given for recurrence of $\mathrm{VH}$ at 5 months after the first surgery, ocular inflammation in the right eye was resolved. However, BCVA remains at $20 / 200$ due to disruption of retinal outer layers in the macula besides retinal ischemic region presented by hyperreflective image in retinal inner layers (Figure 2B).

\section{Discussion}

Although patients with TB-associated uveitis present with a wide spectrum of ocular findings, ${ }^{5}$ CRVO is rare in TBassociated uveitis and only a few cases have been reported. ${ }^{1-3}$ Subclinical granulomas in the optic disc and periphlebitis of the central retinal vein are presumably caused by active ocular inflammation associated with $\mathrm{TB}$, and both require anti-TB therapy. IVR improves vascular permeability and reduces progression of retinal nonperfusion in CRVO. ${ }^{4}$ There were only two case reports on anti-VEGF therapy for TB-associated uveitis. ${ }^{2,6}$ In one case, intravitreal injection of anti-VEGF mAb
A

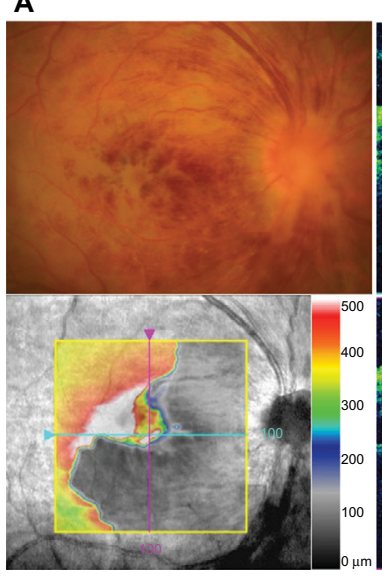

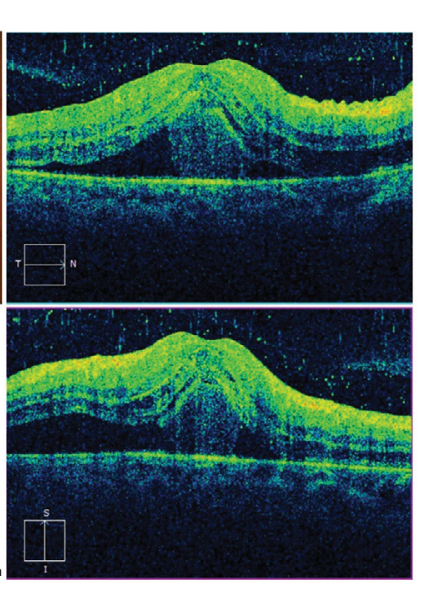

B

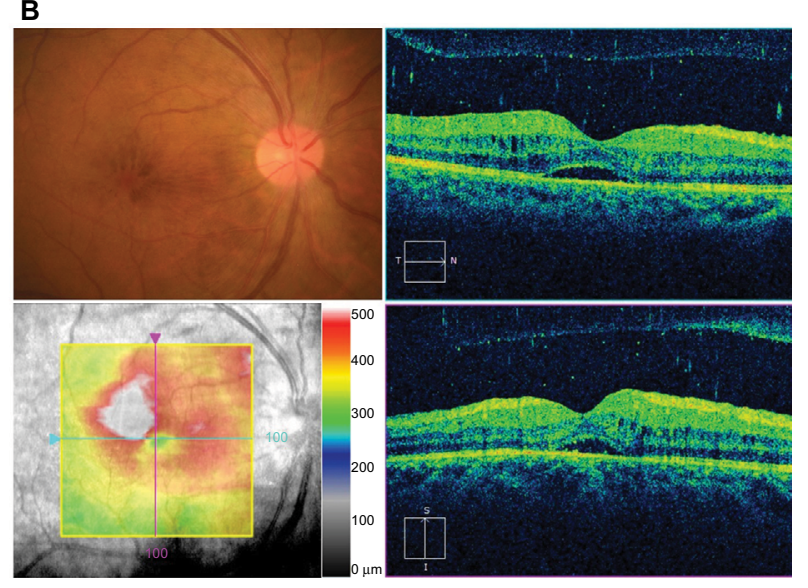

Figure I Comparison of fundus and spectral-domain optical coherence tomography images before and after intravenous injection of ranimizumab (IVR) as an adjunct to anti-TB therapy.

Notes: (A) Fundus findings and macula edema associated with CRVO were observed before IVR and anti-TB therapy. (B) CRVO and macular edema were significantly improved after two sessions of IVR.

Abbreviations: CRVO, central retinal vascular occlusion; IVR, injection of ranimizumab; TB, tuberculosis. 
A

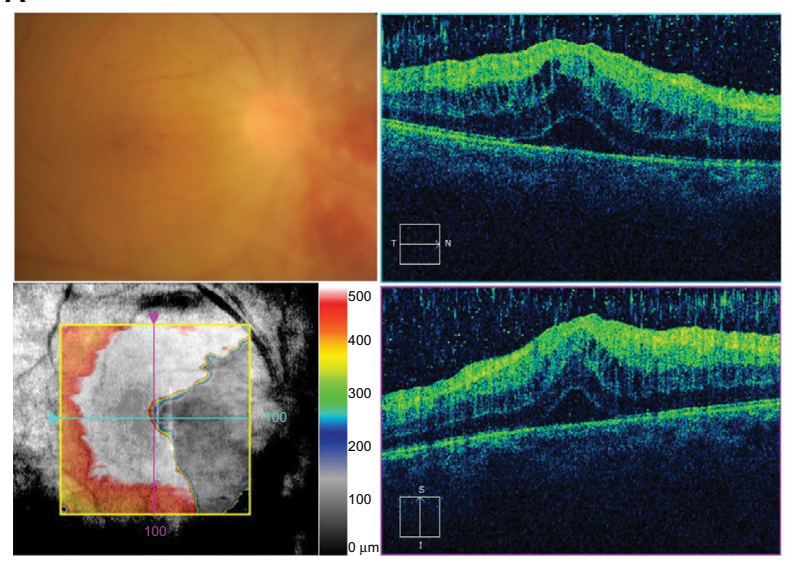

B

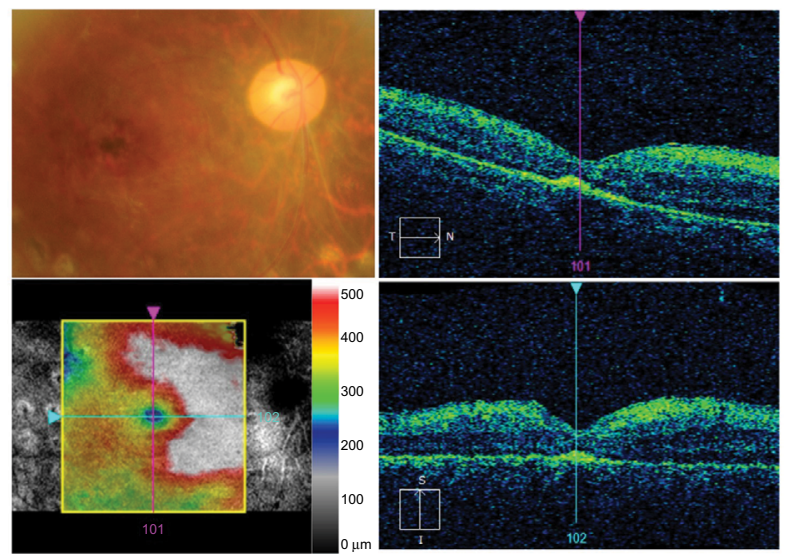

Figure 2 Comparison of fundus and spectral-domain optical coherence tomography images before and after vitrectomy with retinal panretinal photocoagulation.

Notes: (A) Before vitrectomy, retinal hemorrhage in the nasal inferior side with vitreous hemorrhage and relapse of macular edema was detected. (B) After two sessions of vitrectomy with panretinal photocoagulation, retinal hemorrhage and macular edema were resolved, but damage of retinal outer layers in the macula was present.

as an adjunct to anti-TB therapy markedly reduced a granulomatous lesion at the optic disc. ${ }^{6}$ In another case, ${ }^{2}$ however, the treatment was not effective for CRVO in TB-associated uveitis, leading to progression of $\mathrm{VH}$ as in the present case.

\section{Conclusions}

TB-associated uveitis presents periphlebitis, occasionally causing CRVO. IVR is an effective treatment for CRVO; however, it is not promised for CRVO by TB-associated uveitis. Panretinal photocoagulation for retinal nonperfusion area in TB-associated uveitis should be performed for preventing neovascularization that may cause $\mathrm{VH}$. The role of panretinal photocoagulation would not be replaced by anti-VEGF therapy.

\section{Acknowledgments}

Written informed consent was obtained from the patient for publication of this Case Report and accompanying images.

\section{Disclosure}

The authors report no conflicts of interest in this work.

\section{References}

1. Mahyudin M, Choo MM, Ramli NM, Omar SS. Ocular tuberculosis initially presenting as central retinal vein occlusion. Case Rep Ophthalmol. 2010;1(1):30-35.

2. Yuksel E, Ozdek S. Unusual presentation of ocular tuberculosis: multiple chorioretinitis, retinal vasculitis and ischaemic central retinal vein occlusion. Clin Exp Optom. 2013;96(4):428-429.

3. Fullerton DG, Shrivastava A, Munavvar M, Jain S, Howells J, Macdowall P. Pulmonary tuberculosis presenting with central retinal vein occlusion. Br J Ophthalmol. 2007;91(12):1714-1715.

4. Mir TA, Kherani S, Hafiz G, et al. Changes in retinal nonperfusion associated with suppression of vascular endothelial growth factor in retinal vein occlusion. Ophthalmology. 2016;123(3):625.e621-634.e621.

5. Suzuki Y, Suzuki K, Yokoi Y, Miyagawa Y, Metoki T, Nakazawa M. Effects of intravitreal injection of bevacizumab on inflammatory cytokines in the vitreous with proliferative diabetic retinopathy. Retina. 2014;34(1): 165-171.

6. Invernizzi A, Franzetti F, Viola F, Meroni L, Staurenghi G. Optic nerve head tubercular granuloma successfully treated with anti-VEGF intravitreal injections in addition to systemic therapy. Eur J Ophthalmol. 2015;25(3): 270-272.

\section{Publish your work in this journal}

The International Medical Case Reports Journal is an international, peer-reviewed open-access journal publishing original case reports from all medical specialties. Previously unpublished medical posters are also accepted relating to any area of clinical or preclinical science. Submissions should not normally exceed 2,000 words or

\section{Dovepress}

4 published pages including figures, diagrams and references. The manuscript management system is completely online and includes a very quick and fair peer-review system, which is all easy to use. Visit http://www.dovepress.com/testimonials.php to read real quotes from published authors. 